\title{
Electron Scattering from Polarized Deuterium at VEPP-3
}

C.E. Jones, R.J. Holt $\ddagger$, R. Kowalczyk, M. Poelker, D.H. Potterveld, L. Young Argonne National Laboratory, Argonne, Mlinois USA

S.I. Mishnev, D.M. Nikolenko, S.G. Popov, I.A. Rachek, A. Sukhonov, D.K. Toporkov, E.P. Tsentalovich, A. Volosov

Budker Institute for Nuclear Physics, Novosibirsk, Russia

E.R. Kinney

Univ. of Colorado, Boulder, Colorado USA

K.P. Coulter

Univ. of Michigan, Ann Arbor, Michigan USA

C.W. de Jager, G. Retzlaff $\dagger$, J. Theunissen, and H. de Vries NIKHEF-K, Amsterdam, The Netherlands

R. Gilman

Rutgers Univ., Piscataway, New Jersey USA

V.V. Nelyubin

St. Petersburg Institute for Nuclear Physics, Gatchina, Russia

V.N. Stibunov

Tomsk Polytechnic Institute

\begin{abstract}
The status, results, and future plans for the experiment measuring the tensor analyzing power of the deuteron using a tensor-polarized internal target at the VEPP-3 electron storage ring in Novosibirsk are presented.
\end{abstract}

\section{THEORETICAL OVERVIEW}

Elastic electron scattering from deuterium provides information about the electromagnetic structure of the deuteron, which is characterized by three form factors, the charge form factor $F_{C}$, the quadrupole form factor $F_{Q}$, and the magnetic form factor $F_{M}$. In unpolarized elastic electron-deuteron scattering the charge and quadrupole contributions are not experimentally separable. However, this limitation is removed with the introduction of spin observables. Elastic scattering of unpolarized electrons from tensor polarized deuterium is sensitive to the ratio of the form factors, and measurements of $T_{20}$, or correspondingly of the tensor polarization of the outgoing deuterons using an unpolarized target, have been used to separate the charge and quadrupole form factors. $T_{20}$ depends upon the deuteron form factors as

$$
T_{20}=-\sqrt{2}[x(x+2)+y / 2] /\left[2\left(x^{2}+y\right)+1\right]
$$




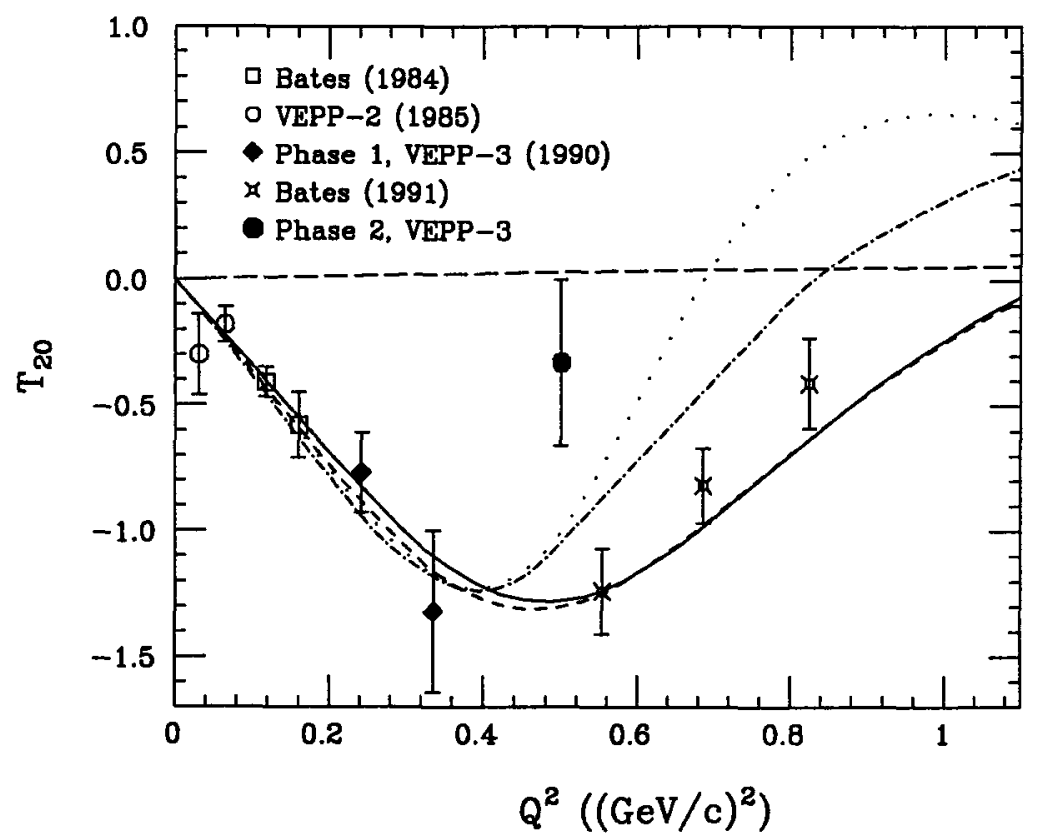

Figure 1: Models of the tensor analyzing power in elastic $e-d$ scattering calculated by Schiavilla and Riska (dotdash) [1], Blunden et al. (dot) [2], Chung et al. (short dash) [3], Hummel and Tjon (solid) [4], and Brodsky and Hiller (long dash) [5]. Also shown are data from previous measurements $[6,7,8,9]$ and the results from the first data run of the second phase of the internal target experiment at VEPP-3.

where $x=\frac{2}{3} \tau\left(F_{Q} / F_{C}\right), y=\frac{\tau}{3}\left(1+2(1+\tau) \tan ^{2}(\theta / 2)\right)\left(F_{M} / F_{C}\right)^{2}$, and $\tau=Q^{2} / 4 m_{d}^{2}$.

Above $Q^{2} \sim 0.40(\mathrm{GeV} / \mathrm{c})^{2} T_{20}$ can be very sensitive to the model used in the calculations because in the impulse approximation the first minimumin $F_{C}$ occurs in this kinematic region and the position of the zero shifts significantly with the introduction of isoscalar meson exchange currents [1]. Fig. 1 shows $T_{20}$ calculated with various theoretical models; the dot-dash line is the calculation of Schiavilla and Riska using the Argonne V14 potential, which includes meson exchange currents explicitly [1]; the dotted line is the coupled channel calculation (model $\mathrm{D}^{\prime}$ ) of Blunden et al. [2]; the short dashed line is the relativistic calculation of Chung et al. using light-front dynamics to calculate the deuteron wave function from a nonrelativistic potential [3]; the solid line is the relativistic calculation of Hummel and Tjon using a covariant wave function calculated from the one-boson exchange model [4]; and the long dashed line is a calculation based on perturbative QCD by Brodsky and Hiller [5]. Also shown are data from previous measurements $[6,7,8,9]$. Data above $Q^{2} \sim 0.40(\mathrm{GeV} / \mathrm{c})^{2}$ can help to constrain models of the deuteron wave function, the effect of non-nucleonic degrees of freedom and the size of relativistic corrections. As data at higher momentum becomes available, one can also test calculations based upon quark degrees of freedom and ultimately test QCD.

It is interesting to note that experimental data for the isoscalar charge form factor of the 3-body system [10] agree with calculations that include isoscalar meson exchange 
currents [11] and disagree with those that assume the plane wave impulse approximation (PWLA). This contradicts the experimental data on $F_{C}$ of the deuteron [9], which is in better agreement with the PWLA than with a model including isoscalar meson exchange currents that is consistent with the 3-body experimental data [1]. Indeed, this phenomenon is not simply a feature of one calculation; Sauer and Henning [12] have shown a linear correlation between the location of the minimum in the deuteron charge form factor and the minimum in the isoscalar 3-body charge form factor, and at present there is no model of the deuteron that both agrees with the deuteron experimental data and, when used as input for three-body calculations, successfully describes the existing data on the three-body isoscalar form factors. Ultimately the two and three body systems must be described within a consistent model. This discrepancy provides impetus for both more theoretical work and experimental confirmation of the position of the first minimum in both the two and three body isoscalar charge form factors.

\section{THE VEPP-3 EXPERIMENT}

The second phase of the experiment to measure $T_{20}$ at the VEPP-3 electron storage using an tensor polarized internal deuterium target and $2 \mathrm{GeV}$ electrons is currently underway. Tensor polarized deuterium from an atomic beam source feed an active storage cell, which can be opened and closed for filling the storage ring. The detector system has two electron arms and two hadron arms arranged symmetrically around the beamline. The arms are nearly identical and provide redundancy to minimize systematic errors. The electron arm covers the angular range of $20^{\circ}-30^{\circ}$ and the hadron arm covers the range $60^{\circ}-70^{\circ}$. In addition, there are two neutron arms in the backward direction $\left(132^{\circ}-138^{\circ}\right)$. Both elastic and inelastic scattering data are collected.

Three data runs have been taken during Phase 2. During the first run, in Jan. '92 June '92, $150 \mathrm{kC}$ of charge were collected with a target thickness of $2.0 \pm 0.5 \times 10^{12} / \mathrm{cm}^{2}$. The beam current was limited by high background rates from beam halo. Before the second run, in Nov. '92 - May '93, collimators were installed upstream of the target region which reduced the background rate by a factor of $\sim 5-10$ in the detectors in both electron and hadron arms. Problems with the source and the detectors in the second run severely limited the useful data collected. At the end of the second run, a low- $\vec{q}$ polarimeter was installed to directly monitor the target polarization using small angle $e-d$ elastic scattering. Analysis of the data from the third run, started in Feb. ' 94 , is not complete.

The third phase of the experiment will use a high density, laser-driven polarized deuterium source coupled to a passive storage cell. Flow rates of up to $1.1 \times 10^{18} \mathrm{~s}^{-1}$ have been demonstrated with the laser-driven source, so it is likely that the target thickness in Phase 3 will be limited by the vacuum requirements of the storage ring. Using the laser-driven source, a target thickness of $1-4 \times 10^{14} \mathrm{~cm}^{-2}$ is anticipated.

\section{RESULTS AND CONCLUSIONS}

The value of $T_{20}$ extracted from data collected in the first run of the current phase of the experiment is shown as a solid circle in Fig. 1. Data (not shown) collected simultaneously at lower $Q^{2}$ was normalized to the Paris potential PWIA calculation 
to determine the average target tensor polarization. The error bar on the data point shown includes the uncertainty from the polarization determination. The normalization procedure yields $p_{z z}=0.67 \pm 0.19$, which is in agreement with the value of $p_{z z}=$ $0.58 \pm 0.17$ obtained with the low $\vec{q}$ polarimeter at the end of the second data run.

The statistical accuracy of the data from Phase 2 of the VEPP-3 experiment is not yet sufficient to indicate the need for isoscalar meson exchange currents, although the point lies above the PWIA calculations and the previous data in this kinematic region. Potentially, the measurements at VEPP-3 provide an important verification of the existing data because the internal target technique is subject to different systematic errors than the recoil polarimeter technique used in the Bates measurement. Better data in this region, including the data at higher $Q^{2}$ to be taken in Phase 3 of the experiment where it is possible to extend the measurements to $Q^{2}=1(\mathrm{GeV} / \mathrm{c})^{2}$, may shed light on the existing discrepancy between the calculations of the two and three body system.

This work is supported in part by the U.S. Department of Energy, Nuclear Physics Division, under contract W-31-109-ENG-38, the Russian Fund for Fundamental Research, the International Science Foundation, Grant N RBC000, and the Netherlands' Organization for Scientific Research, under contract 713-119.

\section{REFERENCES}

[t] Present address: Physics Dept., Univ. of Ilinois, Urbana, Illinois.

[t] Present address: Saskatchewan Accelerator Laboratory, Saskatoon, Canada.

1. R. Schiavilla and D. O. Riska, Phys. Rev. C 43, 437 (1991).

2. P. G. Blunden et al., Phys. Rev. C 40, 1541 (1989).

3. P. L. Chung et al., Phys. Rev. C 37, 2000 (1988).

4. E. Hummel and J. A. Tjon, Phys. Rev. Lett. 63, 1788 (1989).

5. Stanley J. Brodsky and John R. Hiller, Phys. Rev. D 46, 2141 (1992).

6. M. E. Schulze et al., Phys. Rev. Lett. 52, 597 (1984).

7. V. F. Dmitriev et al., Phys. Lett. 157B, 143 (1985).

8. R. Gilman et al., Phys. Rev. Lett. 65, 1733 (1990).

9. I. The et al., Phys. Rev. Lett. 67, 173 (1991).

10. A. Amroun et al., Phys. Rev. Lett. 69, 253 (1992).

11. R. Schiavilla, V. R. Pandharipande, and D. O. Riska, Phys. Rev. C 41, 309 (1990).

12. P. U. Sauer and Hartmut Henning, in Few Body Problems in Physics '93, ed. B. I G. Bakker and R. van Dantzig (Springer-Varlag, Vienna, 1994). 